Original Paper

\title{
PETSTEP: Generation of synthetic PET lesions for fast evaluation of segmentation methods
}

\author{
Beatrice Berthon ${ }^{\mathrm{a}, *}$, Ida Häggström ${ }^{\mathrm{b}}$, Aditya Apte ${ }^{\mathrm{c}}$, Bradley J. Beattie ${ }^{\mathrm{c}}$, Assen S. Kirov ${ }^{\mathrm{c}}$, \\ John L. Humm ${ }^{c}$, Christopher Marshall ${ }^{a}$, Emiliano Spezi ${ }^{d}$, Anne Larsson ${ }^{b}$, \\ C. Ross Schmidtlein ${ }^{\mathrm{c}}$ \\ a Wales Research E' Diagnostic PET Imaging Centre, Cardiff University, Cardiff, Wales, UK \\ ${ }^{\mathrm{b}}$ Department of Radiation Sciences, Umeå University, Umeå, Sweden \\ c Department of Medical Physics, Memorial Sloan Kettering Cancer Center, New York, USA \\ d School of Engineering, Cardiff University, Cardiff, Wales, UK
}

\section{A R T I C L E I N F O}

\section{Article history:}

Received 26 May 2015

Received in revised form 7 July 2015

Accepted 8 July 2015

Available online 28 August 2015

\section{Keywords:}

Positron emission tomography

Digital phantoms

Simulation

Image segmentation

Synthetic lesions

\begin{abstract}
A B S T R A C T
Purpose: This work describes PETSTEP (PET Simulator of Tracers via Emission Projection): a faster and more accessible alternative to Monte Carlo (MC) simulation generating realistic PET images, for studies assessing image features and segmentation techniques.

Methods: PETSTEP was implemented within Matlab as open source software. It allows generating threedimensional PET images from PET/CT data or synthetic CT and PET maps, with user-drawn lesions and user-set acquisition and reconstruction parameters. PETSTEP was used to reproduce images of the NEMA body phantom acquired on a GE Discovery 690 PET/CT scanner, and simulated with MC for the GE Discovery LS scanner, and to generate realistic Head and Neck scans. Finally the sensitivity (S) and Positive Predictive Value (PPV) of three automatic segmentation methods were compared when applied to the scanner-acquired and PETSTEP-simulated NEMA images.

Results: PETSTEP produced 3D phantom and clinical images within 4 and 6 min respectively on a single core $2.7 \mathrm{GHz}$ computer. PETSTEP images of the NEMA phantom had mean intensities within $2 \%$ of the scanner-acquired image for both background and largest insert, and 16\% larger background Full Width at Half Maximum. Similar results were obtained when comparing PETSTEP images to MC simulated data. The S and PPV obtained with simulated phantom images were statistically significantly lower than for the original images, but led to the same conclusions with respect to the evaluated segmentation methods. Conclusions: PETSTEP allows fast simulation of synthetic images reproducing scanner-acquired PET data and shows great promise for the evaluation of PET segmentation methods.
\end{abstract}

(C) 2015 Associazione Italiana di Fisica Medica. Published by Elsevier Ltd. This is an open access article under the CC BY-NC-ND license (http://creativecommons.org/licenses/by-nc-nd/4.0/).

\section{Introduction}

In Positron Emission Tomography (PET) images, the segmentation of tumor from background has a number of important applications in both prognosis [1,2] and therapy [3,4]. However, implementation of automated segmentation into the clinical environment has been slow primarily due to the lack of standardized means with which to evaluate the various methods [5]. This includes the availability of data with a known ground truth. At present, such data are available on a limited scale and for a very large range of image types, making standardization problematic. The

\footnotetext{
* Corresponding author. Wales Research \& Diagnostic PET Imaging Centre, Cardiff University, room GF705 Ground floor 'C' Block, Heath Park, Cardiff CF14 4XN, UK. Tel.: +44 0292074 2555; fax: +44 (0) 2920746982.

E-mail address: BerthonB@cardiff.ac.uk (B. Berthon).
}

American Association of Physicists in Medicine (AAPM) Task Group- $211^{1}$ (TG-211), Classification and evaluation strategies of autosegmentation approaches for PET, is seeking to establish a methodology and a framework for evaluating auto-segmentation methods. In a forthcoming report, the TG-211 will be highlighting the need for standard evaluation data available to all and containing a large number of varied images for the evaluation of PET autosegmentation tools. In particular, the use of simulated PET images can be beneficial for the evaluation of segmentation methods, as it theoretically allows generating realistic PET images simulated from different lesion uptakes with known ground truth [6]. However, the large variation of observed lesion geometries and uptake distributions requires a large number of test images to provide clinically relevant and robust results. For such applications, there is a need

\footnotetext{
1 http://aapm.org/org/structure/default.asp?committee_code=TG211
} 
for a fast, flexible, and accessible simulation tool dedicated to the generation of large datasets.

Simulated PET images are useful for a number of applications, ranging from equipment calibration and optimization to testing of novel image processing approaches [7]. The advantages of simulation over both physical phantom and patient data include a greater knowledge, control, and flexibility in defining the tracer uptake distribution. Furthermore, it provides the ability to perform these studies without requiring access to a scanner, which can be limited. When simulating PET images with synthetic lesions, Monte Carlo (MC) simulation of the data followed by reconstruction of the image is most commonly used [6,8-12]. However work presented by Manjeshwar et al. has shown that realistic synthetic lesions can be placed in existing PET images by using arbitrary combinations of ellipsoidal primitives [13] that are added, with noise, to the existing projection data. This work extends Manjeshwar et al. by obviating the need for access to the actual patient projection data and the subsequent required knowledge of and use of the associated imaging system's system matrix. Furthermore, this approach sidesteps the vast computation expense that generally accompanies MC methods [8-11], which can often require hundreds of hours of simulation time to recreate a single bed position from a patient's scan, especially when Graphics Processing Units (GPUs) are not available. This study introduces this forward-projection simulation method to the segmentation community in the form of a fast simulation tool for generating simulated PET lesions and images for use in developing and evaluating segmentation methods.

In this work, we present PETSTEP (PET Simulator of Tracers via Emission Projection), a tool that allows the simulation of synthetic PET lesions with a high degree of flexibility, minimal computational time, and intrinsic data/projector matching, and we describe its implementation. We also compare the images provided by our software to both phantom and clinical data obtained from our scanner as well as to MC simulated phantom data. Finally, we evaluate the impact of using images simulated with our software compared to scanneracquired images for the evaluation of segmentation methods.

\section{Methods}

Description of PETSTEP

\section{Generation of synthetic lesions}

PETSTEP allows the generation of synthetic PET images based on inserting a lesion-like sub-image into an image representing the background. The background image may be a reconstructed PET scan of a patient or phantom, or it can be an idealized image ${ }^{2}$ representing the background object prior to its being acted on by the PET system and the subsequent reconstruction. These two cases are described in the following paragraphs.

Inserting synthetic lesions into images of the idealized background. The most straightforward case is to insert an idealized synthetic lesion directly into an idealized background that represents the underlying distribution of tracer uptake. In this case, it is assumed that neither the background image nor the lesion has been operated on by the system's Point Response Function (PRF), and the image is noise free. The simulation process includes the following steps, illustrated on Fig. 1a):

\footnotetext{
2 In this case, the use of idealized image is meant to convey that the activity distribution that is represented by the background image is the discretized expectation value drawn from an underlying probability density function, which can be defined as realistically as desired.
}

1. The lesion is added to or used to replace the background at its location, as specified by the user.

2. The resulting combined-object, representing the idealized background and lesion is blurred to mimic the effect of a real PET system's PRF. In this study we represent this with a spatially shift invariant PRF, commonly referred to as a Point Spread Function (PSF).

3. The blurred image is then forward-projected via a radon transform to produce noise free projection data.

4. The resulting projection data are attenuated by a forwardprojection of the attenuation map derived from the computed tomography (CT) image. The attenuated data are then scaled, so that the sum of the intensities corresponds to the number of true counts being simulated, which are calculated from the userdefined maximum uptake, uptake distribution, scan time and system sensitivity.

5. Random events and scatter are added to the image. The random distribution is generated from a uniform background, whereas the scatter distribution is generated from the forward projection of the blurred image. The random and scatter distributions are scaled to the number of counts corresponding to the user defined scatter $(S F)$ and random $(R F)$ fractions,

$S F=\frac{S}{T+S}, \quad$ and $\quad R F=\frac{R}{T+S+R}$,

where $T$ is the number of true counts, $S$ is the number of scatter counts, and $R$ is the number of random counts.

6. Noise is added to the data as a Poisson distribution of values with mean value corresponding to the forward projected data with added random and scatter counts.

7. The noisy realizations of the projection data can then be reconstructed using filtered back-projection (FBP) or a maximumlikelihood scheme such as ordered-subset expectationmaximization (OSEM). In this study we use an OSEM scheme that allows for PSF correction (this can be generalized to a spatially variant PRF) [14] given by,

$$
f^{k+1,0}=\sum_{j} \frac{f^{k, j-1}}{P S F^{T} * H_{j}^{T} \mu_{j}} P S F^{T} * H_{j}^{T}\left(\frac{g_{j}}{H_{j}\left(f^{k, j-1} * P S F\right)+\frac{R S_{j}}{\mu_{j}}}\right)
$$

where $f^{k}$ is the $k^{\text {th }}$ iteration of the image, $g_{j}$ is the $j^{\text {th }}$ subset of the data, $\mu$ is the attenuation on each projection, $R S$ are the scatter and randoms, $H$ is the forward-projection, $H^{T}$ is the backprojection, and $*$ is the convolution operator between the images and the system's PSF. Note that $f^{0,0}$ is the initial image for the iterative reconstruction: an image of the unit cylinder. For reconstruction without system response the PSF is the delta function.

8. The resulting images can be post-filtered. In this study we use a Gaussian kernel for the transverse plane and 3-point smoothing in the axial direction.

Inserting synthetic lesion(s) into preexisting patient or phantom images. When inserting a lesion into a preexisting PET image, the lesion and background PET image must be treated separately, since the preexisting PET image has already been acted on by an imaging system's PRF, while the lesion has not. This is shown on Fig. 1b).

The background and tumor images are blurred independently. Next, both images are forward-projected and attenuated independently. The number of true counts specified by the user is reflected as the sum of both background and lesion images. However, the scatter and random distributions and counts are determined using the number of counts from the lesion only to avoid adding noise from the background image that is already present in the 

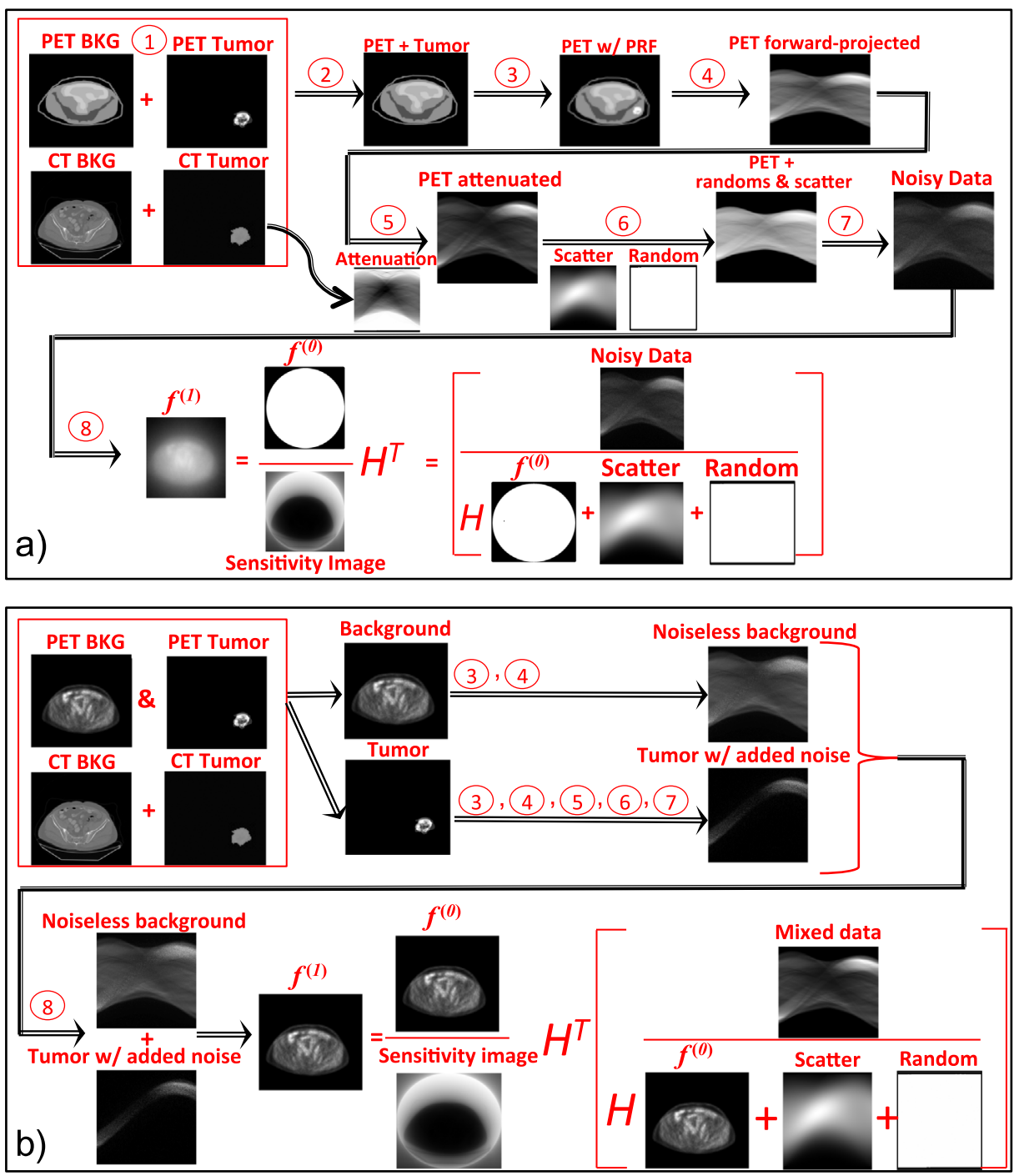

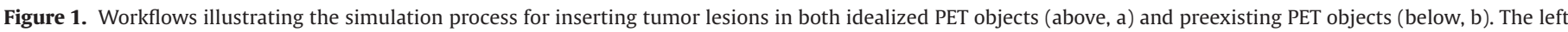

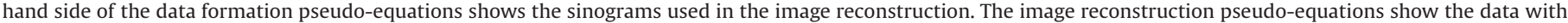
Poisson noise and initializing images for the iterative reconstruction.

preexisting image. The noise realizations are also generated for the lesion sub-image data only. In addition, when reconstructing the data the initializing image $f^{0,0}$ is now the original PET background image. In this way, as each iteration is performed, the only portions of the image that are updated are the lesion and its associated scatter and random noise.

For post-filtering the image, only the inserted lesion and its associated noise are smoothed. This is accomplished by subtracting the preexisting image from the reconstructed one, performing the filtering as described above, and then adding the preexisting image back to the filtered image.

\section{Implementation}

The simulation code is written in Matlab using the radon transform and its adjoint for the forward- and back-projectors, respectively. The PETSTEP package was implemented as a plug-in to the open source software CERR [15].

A user interface was written to allow selecting the parameters for the segmentation. The package relies on the availability of some functions in CERR, in particular the contouring tool for drawing lesion outlines on the scans displayed. PETSTEP requires the presence of one CT scan and one PET scan stored in CERR format, with lesion outlines drawn on the PET image. If several outlines are present, the software adds the corresponding binary masks and multiplies the result by the maximum lesion Standardized Uptake Value (SUV) set by the user. This allows modeling several intensity uptake levels and complex geometric distributions.

The following parameters can be set by the user via a graphical user interface:

- The maximum lesion SUV.

- The blurring filter size in millimeters, corresponding to the scanner's intrinsic resolution.

- The scan time in seconds.

- The background activity concentration in $\mathrm{kBq} / \mathrm{mL}$.

- The count sensitivity in $\mathrm{cps} / \mathrm{kBq}$.

- The scatter and random fractions to be simulated.

- The initial projection data as angular bins and gantry diameter, according to the number of crystals in the scanner and the effective detector diameter.

- The filter size used for PSF correction (the default matches the blurring filter above and is recommended).

- The image size, representing the number of voxels in the transverse plane of the reconstructed image. 
- The number of iterations and subsets for the OSEM reconstruction (the subset number should be a divisor of the angular bins, which is verified by the program).

- The size of the blurring filter applied post reconstruction.

- The type of axial filtering required (3-point smoothing: light, heavy, standard filtering or no axial filtering).

- The number of simulation instances required, corresponding to independent noise realizations.

Additionally, options are provided for the user to:

- Use the lesion uptake data either additively or as a replacement for the original uptake values.

- Use an original PET scan as a ${ }^{18} \mathrm{~F}$-fluorodeoxyglucose (FDG) uptake map as described in the next section.

- Select the type of reconstructions to use.

- Save the PET scans corresponding to the different noise realizations and reconstruction iterations in the current study.

The output of PETSTEP consists of new 3D PET images which are appended to the CERR file from which the simulation was started. These can include:

- The original CT image with inserted lesion density map.

- The original FDG uptake map with inserted lesion, before simulation.

- The simulated PET image for each of the reconstructions selected, and for the number of noise realizations specified.

- The PET scans corresponding to the different noise reconstruction iterations for each reconstruction and noise realization, if specified.

All parameters entered by the user are saved together with each new image generated in CERR. The list of parameters entered can also be saved in a text file in the current folder and retrieved from an existing file.

\section{Evaluation of PETSTEP}

The aim of this investigation was to evaluate the ability of PETSTEP to reproduce realistic FDG PET images for segmentation. For this purpose, we calibrated PETSTEP by determining the set of parameters allowing the closest reproduction of the scanner acquisition and reconstruction process. Work was based on the GE Discovery 690 (D690) PET/CT scanner available at both of our centers (Cardiff and New York) and MC simulations performed with the GATE software using a well validated model of the GE Discovery LS (DLS) PET/CT [16].

\section{Comparison to phantom data}

First, we aimed at reproducing images of the NEMA IEC body phantom acquired previously with a GE D690 PET/CT scanner at our center. The phantom contains six spherical plastic inserts, which were filled with a FDG activity five times higher than the filled-in background activity, and scanned with one bed position. Template images were derived by extracting the phantom geometry from the CT image, and assigning to background and spheres voxel values corresponding to the filled-in activities of the scanned plastic phantom, to model the desired spheres-to-background activity ratio. The scanner specific parameters, such as gantry diameter were set to values obtained from the manufacturer. The number of radial bins and projection angles was derived from the number and size of detector crystals found in the scanner specifications and the reconstructed Field of View (FOV). The system sensitivity was extracted from NEMA NU2 test results published by Bettinardi et al. [17], where the value of 7.5 true $\mathrm{cps} / \mathrm{kBq}$ was obtained for a source length of $700 \mathrm{~mm}$ and was adapted to the modeled detector FOV length of $157 \mathrm{~mm}$, leading to a value of 33.4 true cps/kBq. The scan time and activity concentration (calculated as an average for the whole phantom) were matched to the experimental values. The bed position overlap was set to $50 \%$, to account for axial sensitivity fluctuations across slices, of the D690 scanner, which has 24 detector rings (47 slices per bed position) and an axial coincidence acceptance of \pm 23 slices. The blurring filter size was set to $4.9 \mathrm{~mm}$, which is the average of the PSF Full Width at Half Maximum (FWHM) values of the D690 PET/CT scanner obtained at $1 \mathrm{~cm}$ and $10 \mathrm{~cm}$ of the FOV center using the NEMA NU2 tests obtained at the Cardiff center. The matching PSF correction filter size was set to the same value of $4.9 \mathrm{~mm}$. Scatter and random fractions were also obtained from the NEMA NU2 tests. The OSEM + PSF reconstruction was chosen as the closest to the scanner reconstruction method Vue Point HD algorithm with SharpIR available for the D690 scanner (not including Time-Of-Flight (TOF) correction). The image was reconstructed to a matrix size of $256 \times 256$ to match the matrix size of images from the scanner, with a 3-point axial smoothing filter of [ $\left.\begin{array}{lll}1 & 3 & 1\end{array}\right] / 5$, and post reconstruction filter size of $6.4 \mathrm{~mm}$, matching the filter used for the scanner-acquired image.

The values used for the simulation are summarized in Table 1.

The simulated images (without TOF correction) were compared to the corresponding original scanned PET qualitatively and quantitatively in terms of their intensity spectrum and intensity variations in the background and sphere regions. The total activity in the scan was measured as the sum of all voxel intensities in the three-dimensional (3D) image, multiplied by the voxel volume in $\mathrm{mL}$, as well as the mean intensity value.

The following parameters were estimated for slice No. 14, corresponding to the phantom background only, and for the largest sphere, S6:

- Mean intensity,

- Intensity distribution histogram maximum value,

- Relative (to mean intensity) intensity distribution histogram FWHM.

The background was masked according to its known contour. The values for S6 were calculated within contours generated from the known sphere dimensions and positioned via the high-resolution CT. Histograms were created following the Freedman-Diaconis [18] rule for choosing the bin width and fitted with a Gaussian

Table 1

Parameters used for the simulation of the NEMA IEC body phantom PET scan, for both D690 and DLS scanners, using PETSTEP.

\begin{tabular}{lll}
\hline Parameter name & D690 & DLS \\
\hline CT maximum contrast (\% above background) & 12.5 & 12.5 \\
Maximum SUV & N/A & N/A \\
Blurring filter size $(\mathrm{mm})$ & 4.9 & 5.1 \\
Activity concentration $(\mathrm{kBq} / \mathrm{mL})$ & 5.9 & 4.5 \\
Sensitivity (true cps/kBq) & 33.4 & 42.0 \\
Bed position overlap $(\%)$ & 50 & 31.4 \\
Scan time $(\mathrm{s})$ & 180 & 300 \\
Random fraction & 0.07 & 0.0003 \\
Scatter fraction & 0.37 & 0.40 \\
Radial bins at FOV & $381(700 \mathrm{~mm})$ & $283(550 \mathrm{~mm})$ \\
Projection angles & 288 & 336 \\
Gantry diameter $(\mathrm{mm})$ & 810 & 927 \\
Image matrix size & 256 & 295 \\
Reconstruction type & OSEM + PSF & OSEM \\
Number of iterations & 2 & $8 / 4^{\mathrm{a}}$ \\
Number of subsets & 24 & 12 \\
Post-reconstruction filter size (mm) & 6.4 & 6.0 \\
\hline
\end{tabular}

a 8 iterations for the GATE simulation, 4 iterations for the PETSTEP simulation. 
Table 2

Count statistics obtained for the DLS simulation with GATE and PETSTEP.

\begin{tabular}{ll}
\hline Property & Value \\
\hline Number of trues & $4.13 \times 10^{7}$ \\
Number of randoms & $1.95 \times 10^{4}$ \\
Number of scatters & $2.72 \times 10^{7}$ \\
Total counts & $6.85 \times 10^{7}$ \\
\hline
\end{tabular}

distribution to estimate the mean value and variation (FWHM) of the image intensity in the background and S6.

\section{Comparison to a GATE Monte Carlo reference simulation}

To further validate PETSTEP, the MC software GATE [19] was used together with the previously validated DLS PET camera model [16] to simulate a voxelized representation of the NEMA IEC body phantom, which was simulated with a $5: 1$ ratio of activity concentration in the hot spheres to background. This was compared to a simulation of the phantom using PETSTEP, with the scanner and reconstruction parameters listed in Table 1, to match the DLS model. The DLS PET camera consists of 18 detector rings of 672 BGO crystals each. Each coincidence from the list-mode data was binned [20] into 1) a sinogram matrix of total prompts and 2) a sinogram matrix corresponding to the coincidence type: true, scattered or random. The size of the sinogram matrices was 283 radial and 336 angular bins.

The system sensitivity parameter and phantom activity concentrations used in PETSTEP were adjusted to reproduce the count statistics obtained with the MC simulation given in Table 2 . The prompt coincidences were reconstructed using the software STIR [21] with OSEM (12 subsets, 72 sub-iterations) with normalization, attenuation, scatter and random corrections applied (see Appendices A and B for more details). To account for difference in the reconstruction processes, the simulated PETSTEP image was reconstructed for 1 to 10 iterations (for 12 subsets), and the number of iterations best matching the DLS GATE image, which was reconstructed with 8 iterations, was selected. The images were postfiltered with a $6.0 \mathrm{~mm}$ FWHM Gaussian transverse filter and the 3-point smoothing filter [ $\left.\begin{array}{lll}1 & 2 & 1\end{array}\right] / 4$ in the axial direction. The resulting image was of size $295 \times 295 \times 35$ with a voxel size of $1.97 \times 1.97 \times 4.25 \mathrm{~mm}$.

The variations in the background and largest hot sphere S6 were analyzed in the same manner as with the clinical phantom scan (background slice No. 10), and compared across simulations.

\section{Simulation of realistic clinical data}

PETSTEP, calibrated for the DLS scanner, was used to model realistic head and neck (H\&N) data. A PET uptake map was generated using an available clinical PET/CT scan. This image was manually segmented with CT-based thresholding to separate different anatomical structures. All structures delineated with thresholding were visually checked and manually edited when necessary. A 3D gray level image was generated by assigning a gray level value to each anatomical structure segmented corresponding to its mean intensity on the PET image. The choice of the PET scan and the design of the final template were both validated by a radiologist. The template is shown on Fig. 3b. Normal PET images were simulated from the original CT and uptake template without an added lesion.

In addition, a PET image was simulated from an original H\&N scan, with the insertion of a synthetic lesion using the methodology described above. The simulation parameters used were the same as presented in Table 1, except for the maximum lesion uptake, which was set to 10 times the background uptake.
Finally, a highly heterogeneous lesion was generated by drawing three different overlapping contours in CERR on the H\&N PET uptake map described above, and the simulation was carried out using these contours.

\section{Evaluation of segmentation with PETSTEP}

Finally, we investigated the use of images generated with PETSTEP for the evaluation of PET automatic segmentation (PETAS) methods. Three different PET-AS algorithms described in previous works [22,23] were chosen to represent segmentation approaches found in the recent literature. These included: adaptive iterative thresholding (AT), a gradient-based deformable contouring algorithm (AC) and a fuzzy C-means clustering method for two clusters (FCM2). All PET-AS methods were applied to images of the NEMA phantom acquired with a D690 PET/CT scanner, and to five different noise realizations of simulated images generated in PETSTEP as calibrated to reproduce the D690 scanner (cf. previous section). The segmentation was applied to all six spheres in both images using an initial volume corresponding to a cube centered on the true sphere with $1 \mathrm{~cm}$ margin in all directions. Segmentation results on original and simulated data (averaged over noise realizations) were compared in terms of their spatial conformity by calculating the Sensitivity (S) and Positive Predictive Value (PPV), defined as:

$S=\frac{T P}{T P+F N}$

$P P V=\frac{T P}{T P+F P}$

with TP as the true positives (voxels accurately classified), FN as the false negatives (voxels in true contour not included in segmented contour) and FP as the false positives (voxels in segmentation results not included in true contour).

Statistically significant differences between $S$ and PPV values obtained by the different PET-AS were compared between the two different types of images, and were determined for each PET-AS using the Wilcoxon signed rank test available in SPSS 20 (IBM, Chicago, USA).

\section{Results}

\section{Evaluation of PETSTEP}

\section{Comparison to phantom data}

Figure $2 \mathrm{a}$ and $\mathrm{b}$ provides a comparison of the total activity, background and largest sphere mean intensity and intensity distribution histograms for both D690 original and simulated PET image respectively. The simulated PET image was generated with PESTEP in $1 \mathrm{~min} 23 \mathrm{~s}$, corresponding to $1.8 \mathrm{~s}$ per slice on average on a $2.7 \mathrm{GHz}$ Intel core computer. The number of bins used for background and S6 regions was 135 (bin width $0.06 \mathrm{kBq} / \mathrm{mL}$ ) and 26 (bin width $1.41 \mathrm{kBq} / \mathrm{mL}$ ) respectively, using the average from the Freedman-Diaconis rule. The intensity distribution within S6 was not close enough to be fitted to a Gaussian distribution, and FWHM values are therefore not reported. The total activity measured in the simulated image was within $2 \%$ of the activity in the original non TOF D690 PET scan. The mean intensity in the simulated image was also within $2 \%$ of the original values for both sphere S6 and mean background intensities. The intensity distributions obtained for the background were close, with slightly higher number of counts for the original PET compared to the simulated image (41,045 compared to 32,723 and 187 compared 


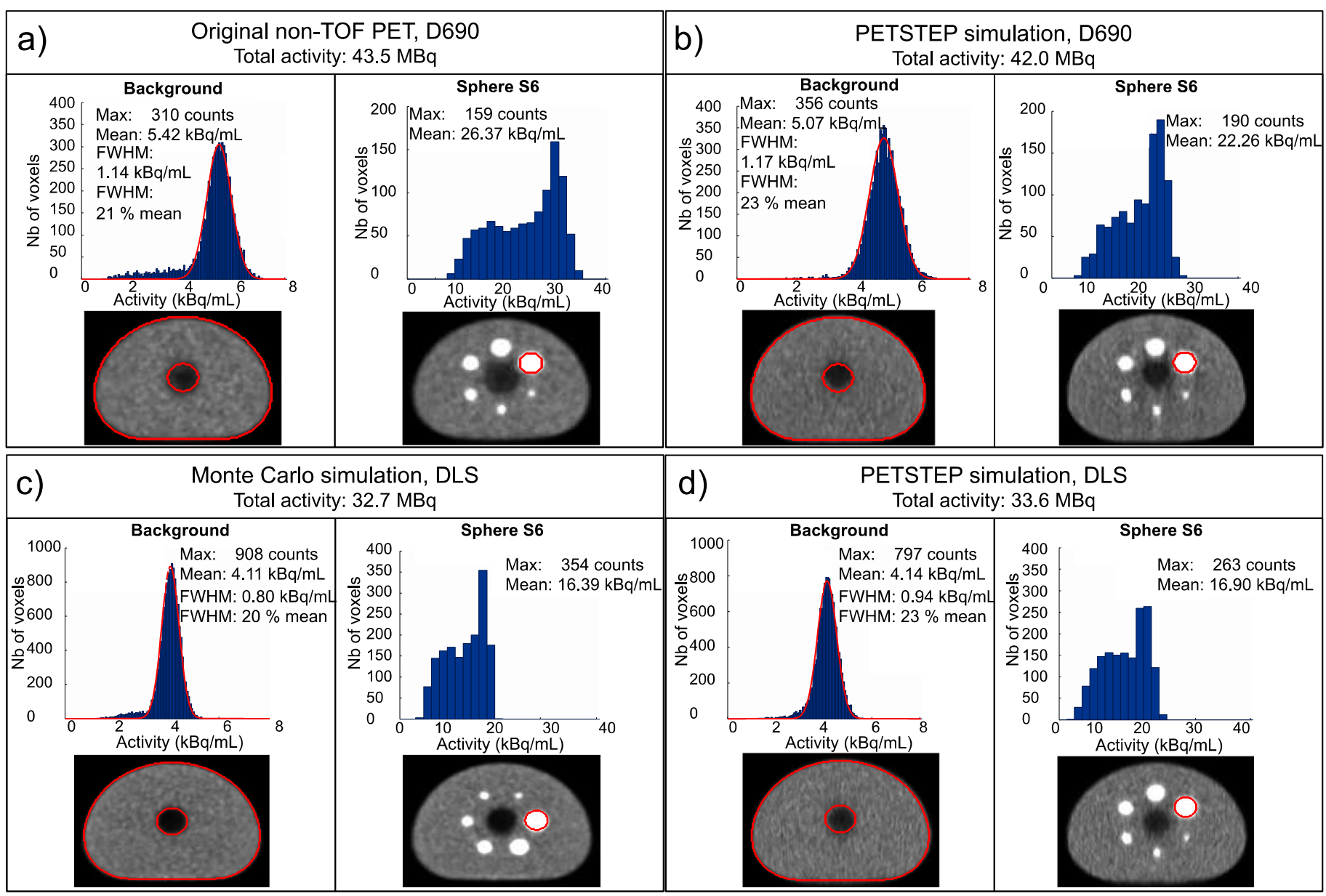

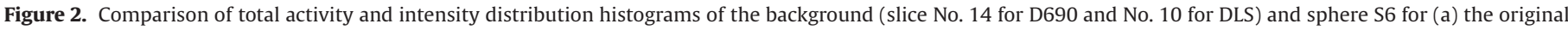

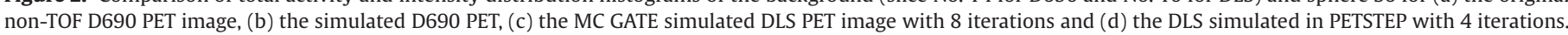

to 164 counts for background and S6 respectively) and larger background FWHM value obtained on the PETSTEP image ( $20 \%$ compared to $17 \%$ of the mean intensity for the original PET).

\section{Comparison to a GATE Monte Carlo reference simulation}

The number of iterations used in PETSTEP to best match data from the GATE MC simulation (reconstructed with 8 iterations) was 4 . The total simulation time was $2750 \mathrm{~h}$ divided over 960 AMD Opteron 6238 (Interlagos) 12-core $2.6 \mathrm{GHz}$ processors. The PETSTEP simulated image was generated in $3 \mathrm{~min}$ and $8 \mathrm{~s}$ on a $2.7 \mathrm{GHz}$ Intel core computer, corresponding to approximately $5.4 \mathrm{~s}$ per slice. The same comparison as for the D690 is shown on Fig. $2 \mathrm{c}$ and $\mathrm{d}$ for the NEMA phantom image acquired with the DLS scanner simulated with MC GATE and with PETSTEP respectively, for the same number of bins as for the GE D690. The total measured activity was $2.5 \%$ higher on the simulated PETSTEP image compared to the GATE simulated image. The mean intensity in the simulated image was within $1 \%$ and $3 \%$ of the original values for the background and sphere S6 respectively. The background FWHMs of the PETSTEP image was slightly larger than for the GATE MC image ( $23 \%$ of the mean value compared to $20 \%$ ), which corresponds to a slightly noisier image shown on the bottom row of Fig. 3.

Preliminary work (see Appendix C) showed that the scatter distribution as modeled in PETSTEP was closest to the corresponding MC simulated scatter distribution for a $20 \mathrm{~cm}$ Gaussian kernel, as determined with a minimum Root Mean Squared Error (RMSE) (see Figs. C1 and C2 in Appendix C).

\section{Simulation of realistic clinical data}

Figure 3 shows a comparison between sagittal slices of the original patient H\&N PET image (panel a), the FDG uptake map extracted from the PET/CT dataset (panel b), and the corresponding simulated PET image (panel c). Observable differences between the original and simulated PET images are located in the nose, tongue and larynx area, for which the image intensity obtained is lower than for the original image. The simulated image was visibly closer to the FDG uptake map. The simulation was completed on a $2.7 \mathrm{GHz}$ Intel core computer in 5 min and $47 \mathrm{~s}$, for a 117-slice image, corresponding to the superior-inferior length of the initial uptake map.

Figure $4 \mathrm{a}$ and $\mathrm{b}$ shows a PET image simulated from an existing PET scan, to which a homogeneous synthetic lesion was added, at a target-to-background ratio of 10 . The new lesion on Fig. $4 \mathrm{~b}$ is visible at the location of the contour drawn on the original PET (Fig. 4a). The lesion measured mean and peak intensities were 8.7 and 10.6 times higher than the background mean intensity measured in a Region of Interest of the same geometry positioned in the soft tissue background. Figure 4c shows the FDG uptake map derived automatically by PETSTEP using the initial background uptake map and the three different overlapping contours. The resulting image is shown on Fig. 4d, with the contour corresponding to the outline of the heterogeneous lesion modeled. 


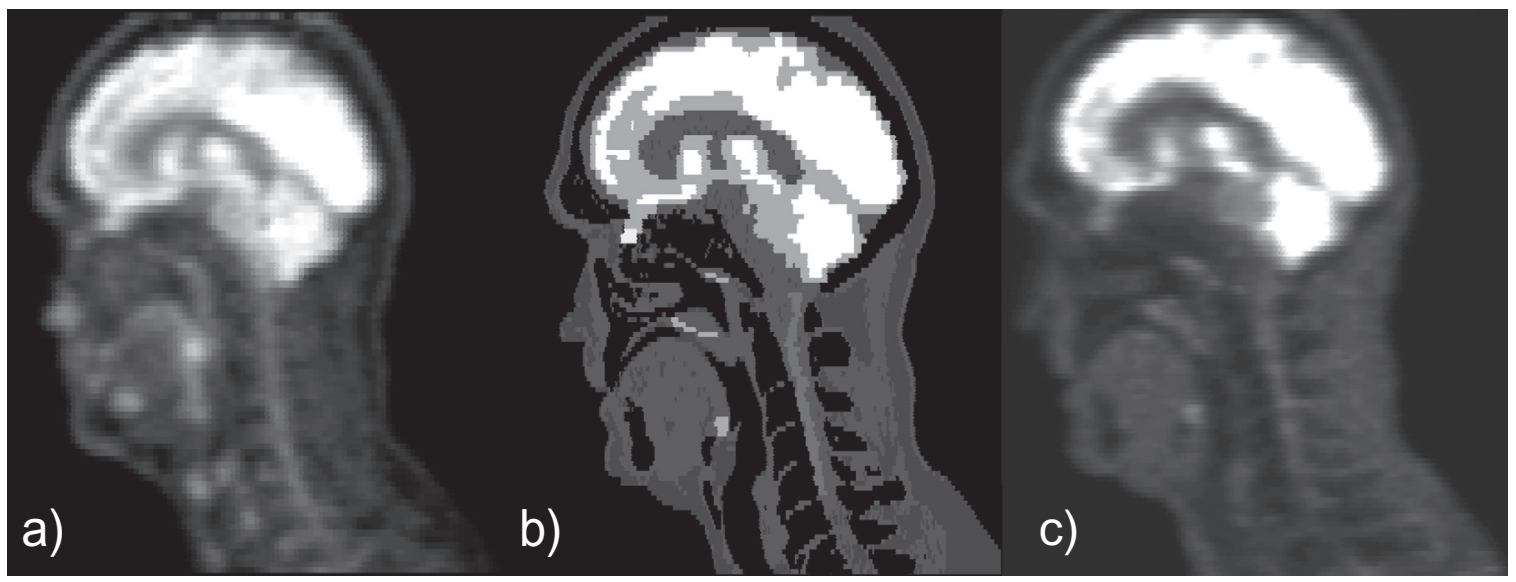

Figure 3. Sagittal slice No. 187 of (a) the original PET image, (b) the FDG uptake map used in the simulation and (c) the simulated PET image.

\section{Evaluation of segmentation with PETSTEP}

Figure 5 shows S values and PPVs obtained for the segmentation of spheres S1 to S6 on PETSTEP and original images by the three PET-AS methods used. Lower S values and PPVs were obtained for PETSTEP simulated images in 16 and 12 out of 18 cases respectively.
Differences in S and PPV were below $24 \%$ and $29 \%$ of the value corresponding to the original PET in all cases. The largest differences (and cases with higher $\mathrm{S}$ for PETSTEP images) were observed for the smallest spheres, for which the standard deviation of values across noise realizations was also the highest (up to $9 \%$ of the value for the original image). FCM2 showed lower S values compared to the

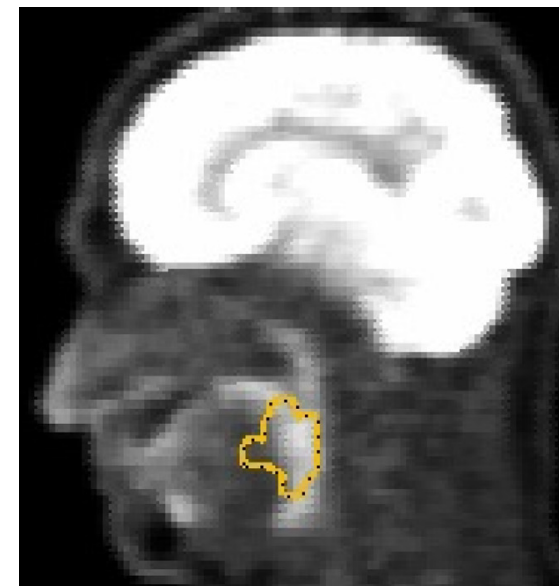

a)

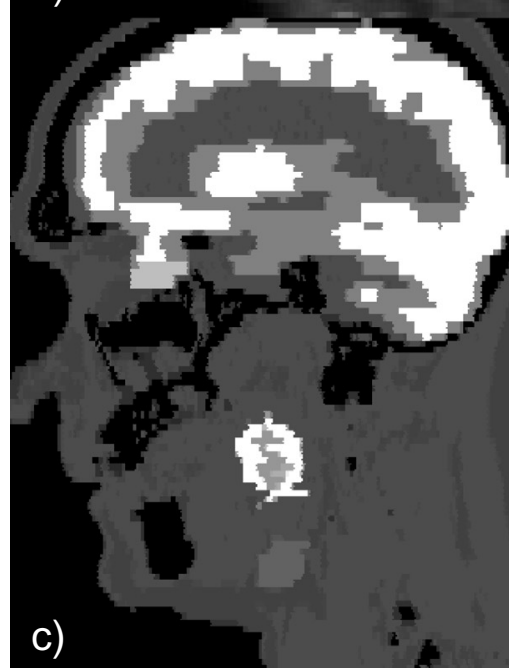

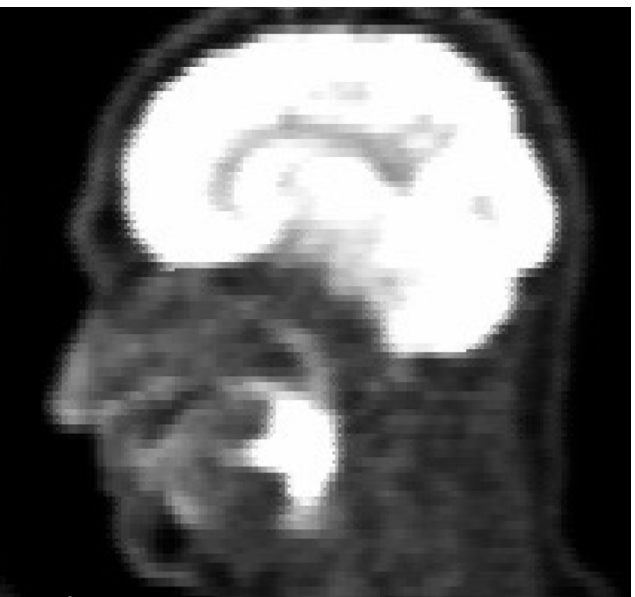

b)

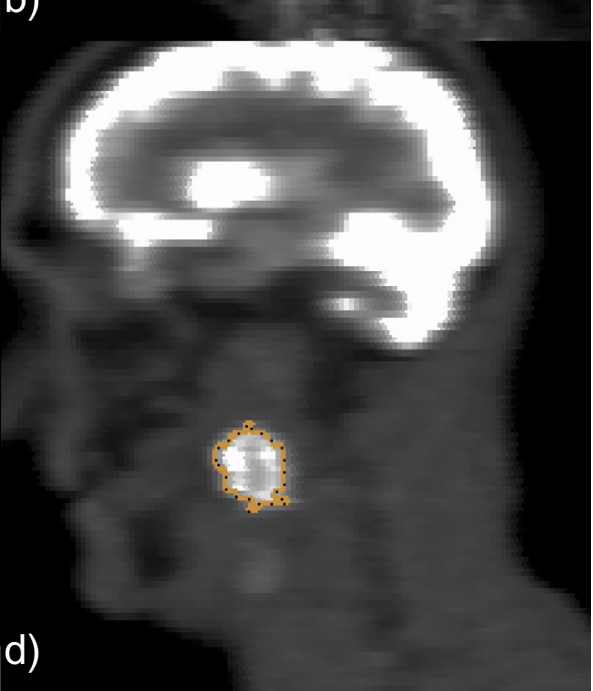

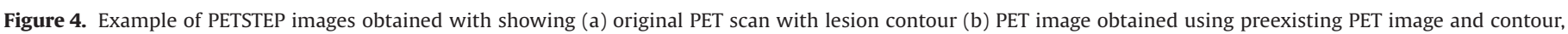
(c) PET uptake map with highly heterogeneous lesion and (d) PET image obtained with lesion contour. 
a)

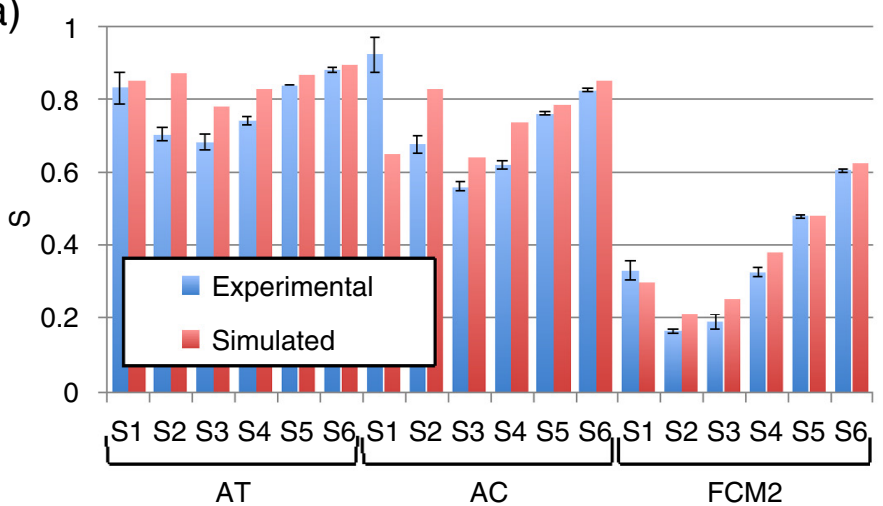

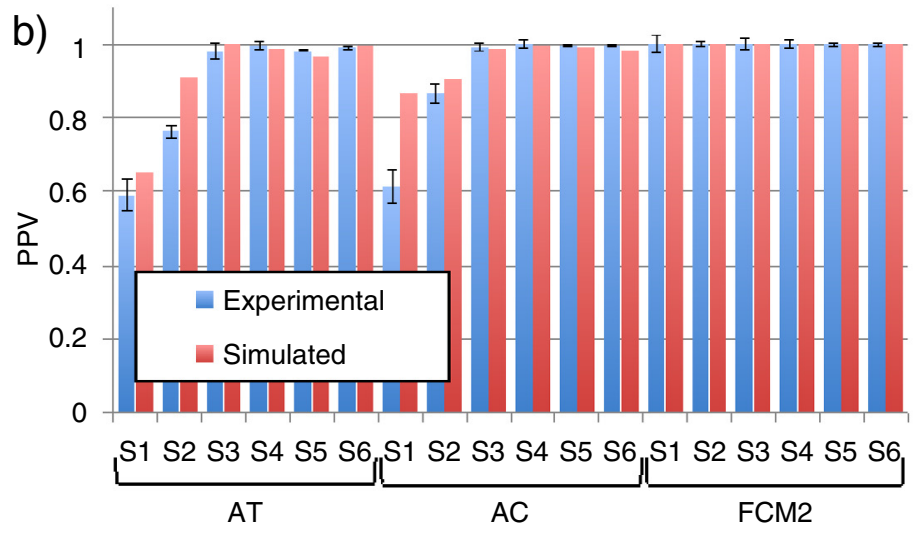

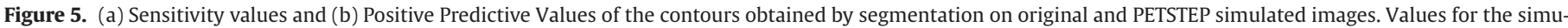
lated case are given as an average on 5 noise realizations, with error bars of one standard deviation of the range of values obtained.

other methods for both types of images, and PPVs of 1 for all spheres were obtained for both image types. Slightly higher $\mathrm{S}$ values were also obtained for AT compared to AC for both image types. Both results obtained with PETSTEP and scanner-acquired images showed higher S value for S1 than S2 and S3 when delineated by FCM2.

The comparison of $S$ values and PPVs obtained by segmenting the simulated (average across simulated instances) and original images showed a significant difference with the Wilcoxon signed rank test $(\mathrm{p}<0.05, \mathrm{Z}=-4.386$ and $\mathrm{p}<0.05, \mathrm{Z}=-2.844$ respectively).

\section{Discussion}

We have developed and implemented PETSTEP, a fast and accessible simulation tool for the generation of synthetic PET images from existing PET as well as from user-defined uptake maps. PETSTEP as implemented as open source uses functionalities already present in CERR and a custom user interface to allow fast simulation of full PET images from complex tracer uptake distributions (FDG in this study), while remaining accessible to users with little or no experience in PET simulation. The addition of synthetic lesions to existing PET images in PETSTEP is similar to work by Manjeshwar et al. (the overlay of freeform shapes versus ellipsoidal primitives) [13]. However, whereas in Manjeshwar et al.'s method tumors are forward-projected and added to the real projection data, PETSTEP forward-projects the existing image to create a synthetic sinogram. To our knowledge, no similar process for PET simulation has been documented in the literature.

PETSTEP involves forward projection with matched projectors, ${ }^{3}$ using the Matlab two-dimensional (2D) radon and unfiltered inverse (adjoint) radon transforms. Modeling of the scanner system is done with filtering and addition of noise distributions. This approach allows matching the simulated data to scanner-acquired images using known measures such as counts, sampling, iterations, etc. This is more clearly seen in the case of maximum-likelihood PET images where the resolution and noise properties are well known to be locally dependent of the imaged objects [8,9]. As a consequence of using this approach, the MC simulation required $2750 \mathrm{~h}$ to generate as many prompts as PETSTEP did in 3 minutes, computing to a factor of $\sim 55,000$ less simulation time. Although recent developments using GPUs can lead to acceleration factors of 400-800 for MC simulations [24], the Radon transform and its adjoint can also

\footnotetext{
3 Matlab's Radon and inverse Radon transforms are matched in the same sense that pixel driven and ray driven projectors are matched. The back projector is not the true adjoint of the forward projector. The use of the inverse Radon transform uses interpolation that makes the match approximate, but close $(\mathrm{O}(\varepsilon)=1 \mathrm{E}-12)$.
}

utilize GPUs for improved performance. This feature will be added to PETSTEP in the near future so that its rapidity compared to MC remains a key advantage. PETSTEP can therefore be extremely useful in the generation of large datasets for quantitative studies and the development of learning methods.

The "inverse crime" model, i.e. the same model is used to generate the data and to reconstruct the images, leads to ignoring normal data inconsistencies due to mismatched acquisition and projectors. However, evidence from Kim et al. [25] shows little effect on image improvement when better system models are used (except for the periphery of the object), which validates the choice of this approach in our work where lesions were at least $3 \mathrm{~cm}$ from the object boundaries. Further limitations of PETSTEP include the absence of correlations between slices that exists in real 3D data. This is accounted for by adjusting the number of counts obtained from the 2D projection data to obtain similar Noise Equivalent Counts (NEC) as measured in 3D. This preserves the effective local NEC in the projection space, so that the appropriate ratios of true, scatter, and random counts are seen by each line of response. In addition, the radon and adjoint radon transforms available in Matlab are defined on a uniformly spaced grid in projection space, and therefore do not match the real detector system spacing. As discussed in the paragraph above the affects of this are small and outweighed by the improvement in image generation speed. The scatter distribution in PETSTEP is modeled using a large Gaussian-blurring kernel of $20 \mathrm{~cm}$ forward-projected into projection space. Since scatter is known [26] to be a slowly varying count distribution dependent on the real source distribution and the attenuation map, realistic local NEC can be achieved by using this underlying distribution in the reconstruction. This approach is justified by the good agreement observed between our scatter model and the MC simulation (cf. Appendix C). However, because of this, PETSTEP is not appropriate for studies specifically investigating or depending on the image scatter distribution. Finally, the attenuation correction is currently performed under the assumption that the whole object or patient scanned has a density equivalent to water. Although the use of the CT image in both simulation and reconstruction should limit the bias added to the image, work is in process to improve this approximation/correction by using a bilinear approach to calculate the attenuation map from the CT image. It should be noted that PETSTEP avoids extensive system modeling, and is therefore designed for evaluating image processing performance rather than true system response. Nevertheless, more advanced image reconstruction schemes and data models can be added easily and work is in progress to do so.

Comparison with phantom images obtained with the D690 PET/ CT scanner have shown that PETSTEP can reproduce the intensity distributions of scanner-acquired non-TOF corrected PET images, with 
intensity distribution means within 3\% (cf. Fig. 2a and b). The slightly higher heterogeneity observed on the simulated images (cf. Fig. 2b) in the background region correlates with a larger FWHM of the intensity distribution histograms. Although the focus of this study was not to reproduce PET images acquired with a specific scanner, such a calibration could be done by applying an additional postreconstruction filter, such as a de-blurring filter to simulate motion correction.

PETSTEP was further used and calibrated to reproduce an image of the NEMA phantom simulated with a MC GATE model of the DLS $\mathrm{PET} / \mathrm{CT}$ scanner. For otherwise matched parameters the number of iterations necessary to reproduce the FWHM of the background obtained for the MC simulation was smaller for PETSTEP reconstruction. It is consistent with the fact that idealized PETSTEP model uses data matched to the projectors and is therefore expected to converge with a smaller number of iterations than MC data reconstructed with STIR. This is a known effect when using "inverse crime" models. However, we additionally note that PETSTEP's convergence was similar to the Discovery 690 PET/CT system, which uses a model that is better matched to the data than STIR. This indicates that our "inverse crime" model for the purposes outlined in this paper is acceptably accurate. Furthermore, even though the MC simulation features a reconstruction algorithm implemented in different software (STIR), the results are still close enough for validation of the scatter model implemented in PETSTEP and to compare image properties.

The flexibility of PETSTEP was demonstrated by reproducing a clinical H\&N scan acquired on the GE D690 and by simulating PET images with both homogeneous and heterogeneous lesions. The H\&N image simulated (cf. Fig. 3c) shows a high degree of similarity with the original PET. Differences in PET intensities in the nose, tongue and vocal cords were due to the fact that the FDG uptake map was designed to represent typical uptake in the resting state. In addition the FDG uptake map was limited to modeling a finite number of tissue structures and of uptake levels in this work. This issue is not observed on Fig. 4b, for which the initial image was an original PET scan. The peak target-to-background ratio measured on the simulated image was within $6 \%$ of the targeted ratio, whereas the mean ratio was $14 \%$ lower, which was expected because of blurring and partial volume effects at the edges of the lesion caused by the simulation process representing the system response and reconstruction. Furthermore, Fig. 4c and d show the ability of PETSTEP to produce images with highly heterogeneous lesion uptake.

The absolute evaluation of the three PET-AS methods for the segmentation of the six NEMA spheres using S and PPV measures provided statistically significantly different results (with the Wilcoxon signed rank test) for images obtained with PETSTEP and original D690 PET images (cf. Fig. 5a and b). Smaller S and PPV were obtained for the PETSTEP images in 16 and 12 out of 18 cases respectively, which could be due to higher noise (background FWHM) observed on the PETSTEP image (cf. Fig. 2). The largest differences in $\mathrm{S}$ values obtained across images, especially for AT and AC, were observed for some of the smallest spheres with higher standard deviation across noise realizations, indicating that these differences may be due to noise fluctuations. AT and AC are indeed based on thresholding and on the image gradient respectively. Both processes are particularly sensitive to noise and intensity fluctuations for small objects. The lower performance (low S and PPV of 1) of FCM2 compared to other methods, observed for the scanneracquired image, was also clearly visible and quantifiable when using PETSTEP data. The superiority of AT to AC seen on scanner-acquired images was confirmed with PETSTEP images. Other small differences in S and PPV observed were too weak to draw any strong conclusions on the relative accuracy of the methods as they were evaluated on a single image. These results show that images simulated with PETSTEP have the potential to provide similar conclusions to scanner-acquired images when used to assess and compare a range of PET-AS approaches. Although PETSTEP may not be capable, at this stage, of providing absolute segmentation results, it could be a very useful tool for the comparison within a same framework of PETAS methods on a variety of complex and realistic data, which would help in addressing the lack of reliable inter-comparisons of PETAS methods on relevant data [27]. This result is important in the light of a growing interest for PET-AS for applications such as RT planning. PETSTEP is currently implemented within the PET-AS set [28] software of the AAPM TG-211 for standardized evaluation of PET auto-segmentation methods.

PETSTEP simulated images also have the potential to be used beyond segmentation evaluation in a number of applications requiring accurate modeling of clinical systems, such as investigations into lesion detection $[29,30]$ or assessment of response to therapy $[31,32]$. Such studies often focus on image processing performance and accurate tracer uptake modeling, thus requiring a large number of images. In addition, PETSTEP can potentially be applied to any given radiotracer, although in the case of cascade photon emitting isotopes the tool will need to be modified. Further, we think that PETSTEP can be a useful starting point for a number of image-based tasks, such as resident education, tumor segmentation and detection, and other machine learning-based processes (radiomics).

\section{Conclusion}

We have developed a fast and flexible PET simulator tool, PETSTEP, for the generation of synthetic PET images representing any userdefined FDG uptake distribution. This tool is open source and designed to be extensible to other isotope and image studies including kinetic modeling. The open source nature of PETSTEP allows user defined uptake distributions that can, in principle, be as complex as desired. We have shown that PETSTEP allows the fast generation of images reproducing scanner-acquired data and can be calibrated to accurately reproduce high quality MC simulated images. We have also shown that PETSTEP provides PET images that can be used for the evaluation of PET segmentation algorithms, providing similar evaluation conclusions to common fillable phantoms. The high flexibility of PETSTEP could further be used for modeling any complex and heterogeneous tracer uptake and for applications such as resident education.

\section{Acknowledgements}

There are no conflicts of interest regarding this work.

The authors thank the AAPM TG-211 for their support, and Dr. J. Fessler for allowing the use of several Matlab statistical packages from his and his student's image reconstruction toolbox.

BB is grateful to Cancer Research Wales for financial support through grant No. 7061. IH would like to thank the Swedish Cancer Society and the Cancer Research Foundation at Umeå University.

\section{Appendix}

\section{A. Scatter correction of Monte Carlo data}

The scatter and random corrections were done by including an additive sinogram in the OSEM loop in STIR. The additive sinogram was created by taking the real scattered and random sinograms and applying filtered back-projection and post-filtering with the same filter as described for the comparison to GATE MC simulation. The images were then forward projected back into projection data and scaled according to the real scatter + random count. 

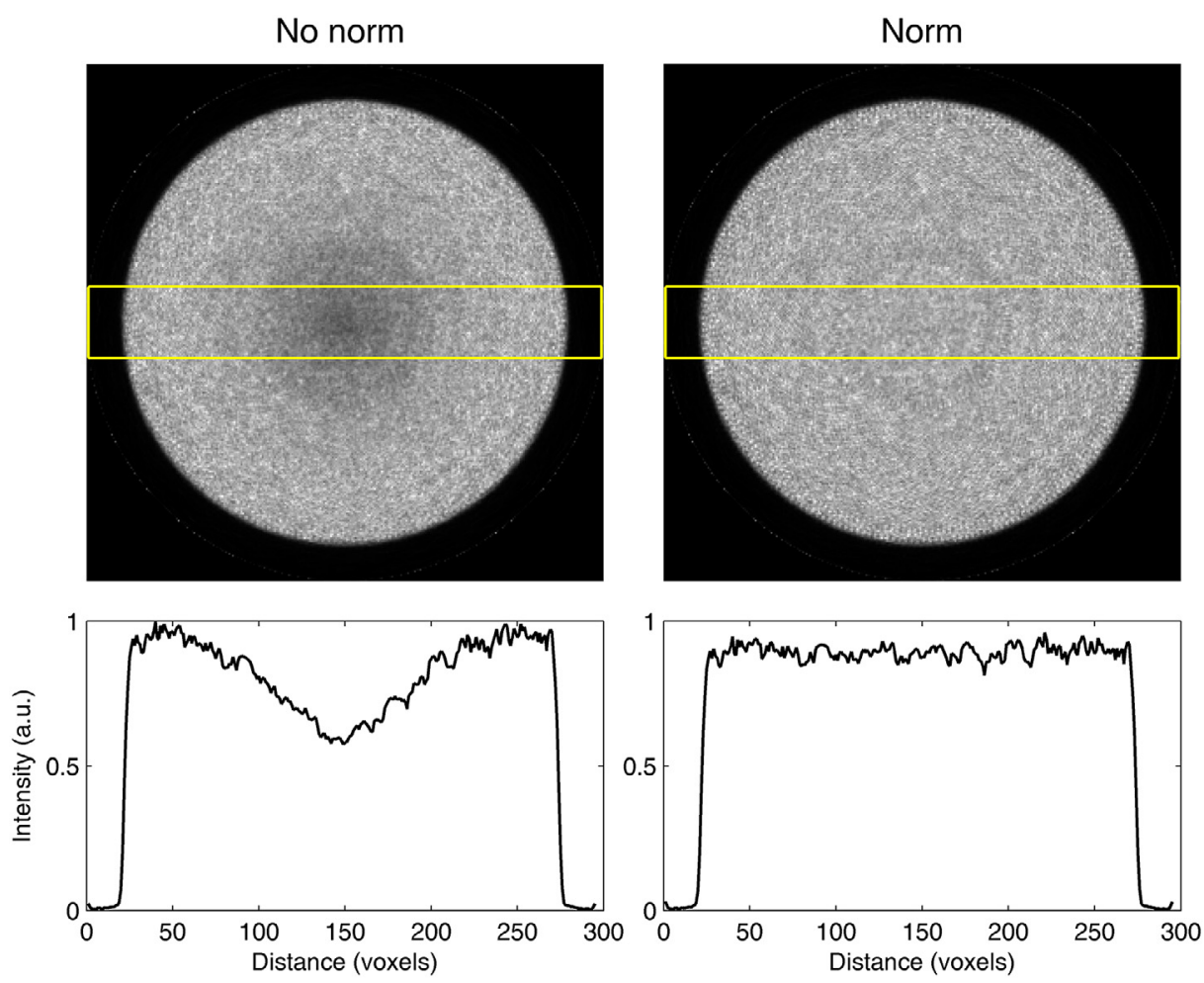

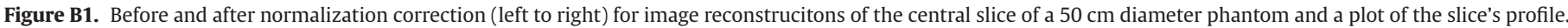

\section{B. Normalization in simulated PET image reconstruction}

In PET image reconstruction, a normalization correction is used to ensure that the data in each line of response are properly weighted to remove the influence of detector response and geometric view factors. Because detector response is generally uniform in most Monte Carlo simulations (it should be noted that GATE does offer non-uniform detector response, however, it is not used here), only the geometric view factors remain. For these simulations, a large cylindrical source without attenuation was used to generate a normalization projection file. This file was used with STIR to perform normalization correction on all image reconstructions. Figure B1 is provided to show the effect of normalization on the image reconstruction of a $50 \mathrm{~cm}$ diameter uniform water cylinder.

The procedure for normalization outlined here was originally implemented in a study by Schmidtlein et al. [16] examining the performance of the GE Discovery ST PET camera, but was here implemented for the GE DLS PET camera. To perform the normalization, a $100 \mathrm{kBq}$ uniform cylindrical source, $59 \mathrm{~cm}$ in diameter by $16 \mathrm{~cm}$ in height, was simulated in a vacuum using a back-to-back photon source for $100,000 \mathrm{~s}$ with decay turned off which resulted in approximately 380 million counts. Several symmetries of the camera

\section{Sinogram Symmetry}

\section{Michelogram Symmetry}

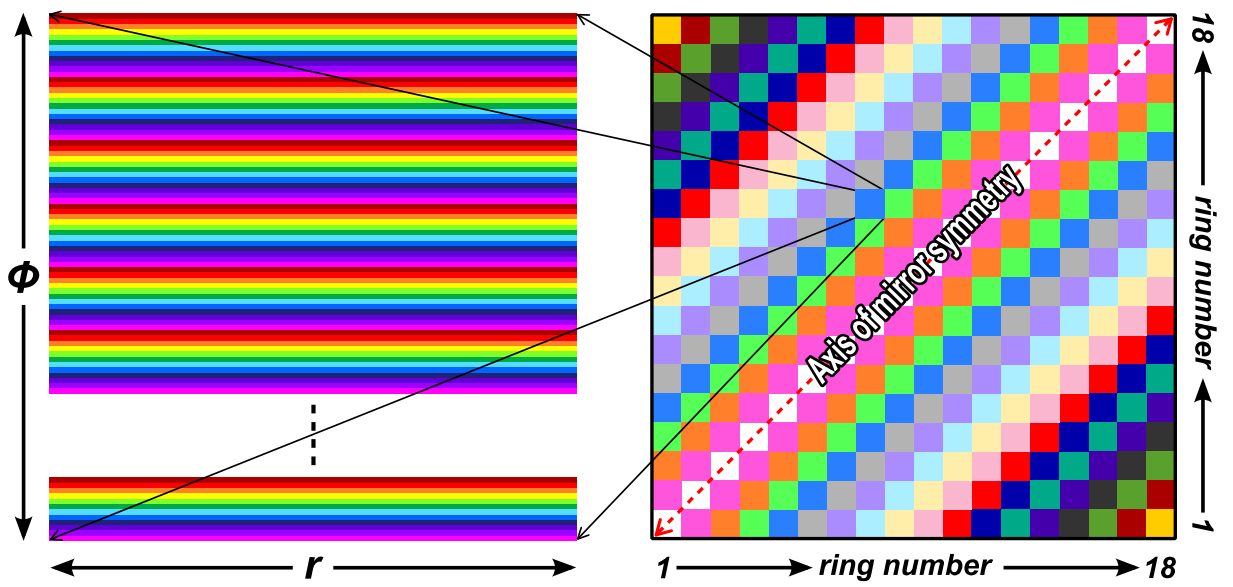

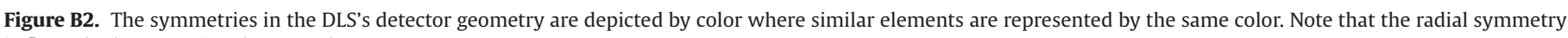
is flipped when crossing the central segment. 


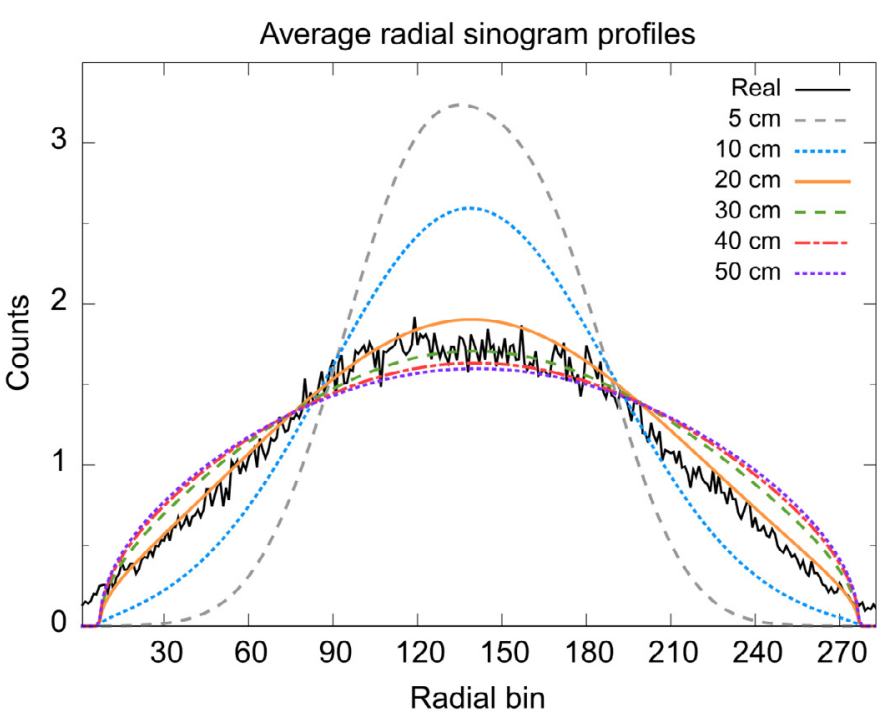

Figure C1. Radial profiles of the direct, central sinograms, averaged over all 336 angular bins. The real profile is obtained from the GATE MC simulation scan and the other 6 from the scatter model with Gaussian kernels of different FWHMs.

were then exploited to amplify the number of effective counts used to create the normalization correction file. These involved the projection angles, the ring differences, and the radial positions. The block structure of the camera repeats itself every 12-projection angles forming a basic symmetry element that was repeated 56 times within each ring difference. If the spacing between the detector blocks in the z-direction is ignored then the geometric response between detectors within each ring difference segment can be assumed to be identical. Furthermore, mirror symmetry in the radial direction between odd and even segments was exploited. Utilizing these symmetries, the number of effective counts increases almost by a factor of 2000, in this case producing 660 billion effective counts.

In addition to exploiting the symmetries, the geometry of the source must also be taken into account. The volume of activity seen by each line of response is a volume given by,

$V_{L O R}(r, \Delta$ ring $)=\sqrt{C(r)^{2}+\Delta \operatorname{ring}^{2}} W_{r} W_{z}$,

where $C(r)$ is the cord length at a particular radial position, $\Delta$ ring is the ring difference spacing, $W_{r}$ is the distance subtended by the detector in the $r$-direction, and $W_{z}$ is the distance subtended by the detector in the $z$-direction. Each bin of the projection file must be normalized with respect to the activity within its FOV. In this case, the activity is proportional to $V_{L O R}(r, \Delta r i n g)$. Figure B2 illustrates these symmetries.

\section{Validation of synthetic PET scatter}

In order to validate the scatter model as implemented in PETSTEP, the same methodology as used for the GE D690 scanner was applied to the GATE simulated data. The voxelized NEMA IEC phantom (noiseless) used as input to GATE was blurred with six Gaussian kernels of different FWHMs: 50, 100, 200, 300, 400, and $500 \mathrm{~mm}$. The resulting images were then forward-projected in STIR and the resulting sinograms were scaled according to the MC prompt count of the scan and the MC simulated scatter fraction. The RMSE was calculated for the difference between the GATE simulated sinogram and the blurred scatter models to evaluate the optimal model.

The comparison of the real scattered coincidences obtained by the GATE MC scan to the true object, blurred by different Gaussians, is seen

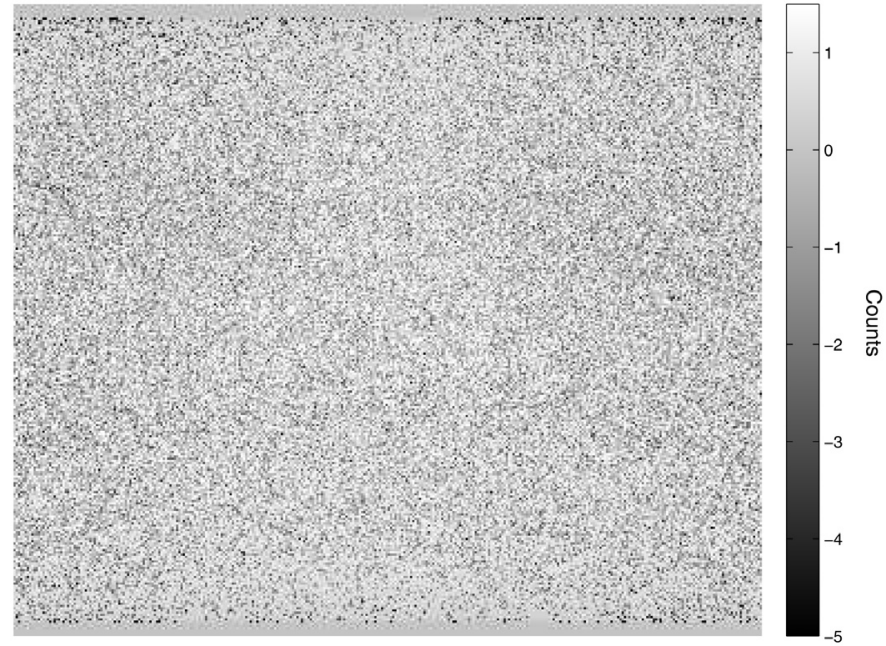

Figure C2. Direct, central sinogram difference (scatter model - real scatters from the GATE simulation), divided by the square root of the model. The scatter model is blurred with a Gaussian of $20 \mathrm{~cm}$ FWHM9.

in Fig. C1. Visual inspection as well as an optimal (minimal) relative RMSE confirmed that the synthetic scatter based on a $20 \mathrm{~cm}$ Gaussian blurring produced the closest fit to the MC data (Figs. C1 and C2).

\section{References}

[1] El Naqa I, Grigsby P, Apte A, Kidd E, Donnelly E, Khullar D, et al. Exploring feature-based approaches in PET images for predicting cancer treatment outcomes. Pattern Recognit 2009;42:1162-71. doi:10.1016/j.patcog.2008.08 011.Exploring

[2] Hatt M, Visvikis D, Pradier O, Cheze-le Rest C. Baseline ${ }^{18}$ F-FDG PET imagederived parameters for therapy response prediction in oesophageal cancer. Eur J Nucl Med Mol Imaging 2011;38:1595-606. doi:10.1007/s00259-011-1834-9.

[3] Paulino AC, Thorstad WL, Fox T. Role of fusion in radiotherapy treatment planning. Semin Nucl Med 2003;33:238-43. doi:10.1053/snuc.2003.127313.

[4] Zaidi H, Vees H, Wissmeyer M. Molecular PET/CT-guided radiation therapy treatment planning. Acad Radiol 2009;16:1108-33.

[5] Lee JA. Segmentation of positron emission tomography images: some recommendations for target delineation in radiation oncology. Radiother Oncol 2010;96:302-7. doi:10.1016/j.radonc.2010.07.003.

[6] Le Maitre A, Segars WP, Marache S, Reilhac A, Hatt M, Tomei S, et al Incorporating patient-specific variability in the simulation of realistic wholebody 18F-FDG distributions for oncology applications. Proc IEEE 2009;97:202638. doi:10.1109/JPROC.2009.2027925.

[7] Hatt M, Cheze le Rest C, Descourt P, Dekker A, De Ruysscher D, Oellers M, et al. Accurate automatic delineation of heterogeneous functional volumes in positron emission tomography for oncology applications. Int J Radiat Oncol Biol Phys 2010;77:301-8. doi:10.1016/j.ijrobp.2009.08.018.

[8] Harrison RL, Vannoy SD, Haynor DR, Gillispie SB, Kaplan MS, Lewellen TK. Preliminary experience with the photon history generator module of a publicdomain simulation system for emission tomography. IEEE Conf Rec Nucl Sci Symp Med Imaging Conf 1993;1154-8. doi:10.1109/NSSMIC.1993.701828.

[9] Ljungberg M, Strand S-E, King MA. The SIMIND Monte Carlo program. In: Monte Carlo calculations in nuclear medicine, second edition: applications in diagnostic imaging. Bristol and Philadelphia: IOP Publishing; 1998. p. 145-63.

[10] Santin G, Strul D, Lazaro D, Simon L, Krieguer M, Vieira Martins M, et al. GATE: a Geant4-Based simulation platform for PET and SPECT integrating movement and time management. IEEE Trans Nucl Sci 2003;50:1516-21.

[11] Espana S, Herraiz JL, Vicente E, Vaquero JJ, Desco M, Udias JM. PeneloPET, a Monte Carlo PET simulation tool based on PENELOPE: features and validation. Phys Med Biol 2009;54:1723-42.

[12] Papadimitroulas P, Loudos G, Le Maitre A, Hatt M, Tixier F, Efthimiou N, et al. Investigation of realistic PET simulations incorporating tumor patient's specificity using anthropomorphic models: creation of an oncology database. Med Phys 2013;40.

[13] Manjeshwar RM, Akhurst T, Beattie BJ, Cline HE, Trotter DE, Gonzalez Humm JL. Realistic hybrid digital phantoms for evaluation of image analysis tools in positron emission tomography. J Nucl Med 2002;43(Suppl. 5):103.

[14] Rapisarda E, Bettinardi V, Thielemans K, Gilardi MC. Image-based point spread function implementation in a fully 3D OSEM reconstruction algorithm for PET. Phys Med Biol 2010;55:4131-51. doi:10.1088/0031-9155/55/14/012. 
[15] Deasy JO, Blanco AI, Clark VH. CERR: a computational environment for radiotherapy research. Med Phys 2003;30:979-85. doi:10.1118/1.1568978.

[16] Schmidtlein CR, Kirov AS, Nehmeh SA, Erdi YE, Humm JL, Amols HI, et al. Validation of GATE Monte Carlo simulations of the GE advance/discovery LS PET scanners. Med Phys 2006;33:198-208. doi:10.1118/1.2089447.

[17] Bettinardi V, Presotto L, Rapisarda E, Picchio M, Gianolli L, Gilardi MC. Physical performance of the new hybrid PET/CT discovery-690. Med Phys 2011;38:5394-411. doi:10.1118/1.3635220.

[18] Freedman D, Diaconis P. On the histogram as a density estimator: L2 theory. Z Wahrscheinlichkeitstheorie Verw Gebiete 1981;57:453-76.

[19] Jan S, Santin G, Strul D, Staelens S, Assi K. GATE: a simulation toolkit for PET and SPECT. Phys Med Biol 2004;49:4543-61.

[20] Schmidtlein C, Turner A, Nehmeh S, Mawlawi O, Erdi Y, Humm J, et al. SU-FFI-54: a Monte Carlo model of the discovery ST PET scanner. Med Phys 2006;33.

[21] Thielemans K, Tsoumpas C, Mustafovic S, Beisel T, Aguiar P, Dikaios N, et al. STIR: software for tomographic image reconstruction release 2. Phys Med Biol 2012;57:867-83. doi:10.1088/0031-9155/57/4/867.

[22] Berthon B, Marshall C, Edwards A, Evans M, Spezi E. Influence of cold walls on PET image quantification and volume segmentation. Med Phys 2013:40:1-13.

[23] Berthon B, Marshall C, Evans M, Spezi E. Evaluation of advanced automatic PET segmentation methods using non-spherical thin-wall inserts. Med Phys 2014;41:022502.

[24] Lemaréchal Y, Bert J, Boussion N, Le Fur E, Visvikis D. Monte Carlo simulations on GPU for brachytherapy applications. IEEE Nucl Sci Symp Conf Rec 2013;5593:doi:10.1109/NSSMIC.2013.6829314.
[25] Kim KY, Kim SM, Lee DS, Lee JS. Comparative evaluation of separable footprint and distance-driven methods for PET reconstruction. J Nucl Med 2013;54:2130.

[26] Valk PE, Bailey DL, Townsend DW, Maisey MN, York N. Positron emission tomography: basic science and clinical practice, vol. 26. New York: SpringerVerlag; 2004 doi:10.1521/aeap.2014.26.2.185.

[27] MacManus M, Nestle U, Rosenzweig KE, Carrio I, Messa C, Belohlavek O, et al. Use of PET and PET/CT for radiation therapy planning: IAEA expert report 2006-2007. Radiother Oncol 2009;91:85-94. doi:10.1016/j.radonc.2008.11.008.

[28] Shepherd T, Berthon B, Galavis P, Spezi E, Apte A, Lee JA, et al. Design of a benchmark platform for evaluating PET-based contouring accuracy in oncology applications. Eur J Nucl Med Mol Imaging 2012;39:S264.

[29] Moore SC, Foley Kijewski M, El Fakhri G. Collimator optimization for detection and quantitation tasks: application to gallium-67 imaging. IEEE Trans Med Imaging 2005;24:1347-56. doi:10.1109/TMI.2005.857211.

[30] Cao N, Huesman RH, Moses WW, Qi J. Detection performance analysis for time-of-flight PET. Phys Med Biol 2010;55:6931-50. doi:10.1088/0031-9155/ 55/22/021.

[31] Pshenichnov I, Larionov A, Mishustin I, Greiner W. PET monitoring of cancer therapy with $3 \mathrm{He}$ and $12 \mathrm{C}$ beams: a study with the GEANT4 toolkit. Phys Med Biol 2007;52:7295-312. doi:10.1088/0031-9155/52/24/007.

[32] Van Velden FHP, Cheebsumon P, Yaqub M, Smit EF, Hoekstra OS, Lammertsma AA, et al. Evaluation of a cumulative SUV-volume histogram method for parameterizing heterogeneous intratumoural FDG uptake in non-small cell lung cancer PET studies. Eur J Nucl Med Mol Imaging 2011;38:1636-47. doi:10.1007/ s00259-011-1845-6. 